\title{
What Are the Results and the Prognostic Factors of Motor Cortex Stimulation in Patients with Facial Pain? A Systematic Review of the Literature
}

\author{
Alessandro Rapisarda ${ }^{a}$ Eleonora loannoni ${ }^{b}$ Alessandro Izzo ${ }^{a}$ \\ Nicola Montano ${ }^{a}$ \\ aDepartment of Neuroscience, Neurosurgery Section, Fondazione Policlinico Universitario Agostino Gemelli IRCCS, \\ Università Cattolica del Sacro Cuore, Rome, Italy; ${ }^{b}$ Department of Anesthesiology and Intensive Care Medicine, \\ Fondazione Policlinico Universitario A. Gemelli IRCCS, Rome, Italy
}

\section{Keywords}

Motor cortex stimulation · Facial pain · Trigeminal neuralgia .

Trigeminal neuropathic pain · Anaesthesia dolorosa .

Systematic review

\begin{abstract}
Introduction: Facial pain (FP) is a type of neuropathic pain which recognizes both central and peripheral causes. It can be difficult to treat because it can often become resistant to pharmacological treatments. Motor Cortex Stimulation (MCS) has been used in selected cases, but the correct indications of MCS in FP have not been fully established. Here we systematically reviewed the literature regarding MCS in FP analysing the results of this technique and studying the possible role of different factors in the prognosis of these patients. Methods: A literature search was performed through different databases (PubMed, Scopus, and Embase) according to PRISMA guidelines using the following terms in any possible combination: "facial pain" or "trigeminal" or "anaesthesia dolorosa" and "motor cortex stimulation." Results: 111 articles were reviewed, and 12 studies were included in the present analysis for a total of 108 patients. Overall, at latest follow-up (FU), $70.83 \%$ of patients responded to MCS. The preoperative VAS significantly decreased at the latest FU $(8.83 \pm 1.17$ and $4.31 \pm 2.05$, respectively; $p<0.0001)$. Younger
\end{abstract}

age $(p=0.0478)$ and a peripheral FP syndrome $(p=0.0006)$ positively affected the definitive implantation rate on univariate analysis. Younger age emerged as a factor strongly associated to a higher probability to go to a definitive MCS implant on multivariate analysis ( $p=0.0415)$. Conclusion: Our results evidenced the effectiveness of MCS in treating FP. Moreover, the younger age emerged as a positive prognostic factor for definitive implantation. Further studies with longer FU are needed to better evaluate the long-term results of MCS.

(c) 2021 S. Karger AG, Basel

\section{Introduction}

Neuropathic pain (NP) can substantially impair quality of life and is often associated with other problems such as loss of function, anxiety, depression, disturbed sleep, and impaired cognition [1]. Standard treatment consists of pharmacological therapies, but this type of pain often shows a tendency to become refractory to different drugs and painkillers [2]. Chronic facial pain (FP) recognizes different causes $[3,4]$ such as cerebrovascular accidents (central post-stroke pain and thalamic pain), different types of trigeminal pain (trigeminal NP, trigeminal deafferentation pain, and post-herpetic neuralgia) and the

karger@karger.com www.karger.com/ene (c) 2021 S. Karger AG, Base

Karger"
Correspondence to:

Nicola Montano, nicolamontanomd@yahoo.it 
atypical FP, recently re-defined as persistent idiopathic FP by the International Headache Society, whose exact physiopathology is still unknown [5]. Motor cortex stimulation (MCS) was firstly advocated as a type of treatment for NP by Tsubokawa et al. who reported a considerable improvement of pain in a patient suffering from central deafferentation pain [6]. Since then, several case reports and case series have been reported on the results of MCS for different types of NP and FP [7-16]. Although a previous critical review about NP suggested the usefulness of MCS in these patients [17] and a meta-analysis showed the effectiveness of MCS over placebo and transcranial magnetic stimulation (TMS) in different NP [18] syndromes, the correct indications of MCS have not been fully established. Moreover, to the best of our knowledge, no systematic review with a pooled analysis of the literature has been published about the results of MCS in FP. The aim of our study was to systematically review the literature regarding MCS in FP analysing the results of this technique in these patients. Furthermore, we studied the possible role of different factors in the prognosis of $\mathrm{FP}$ patients submitted to MCS.

\section{Methods}

Inclusion Criteria and Outcomes Measurement

This study was conducted in agreement with the PRISMA guidelines statement [19]. Three medical databases (PubMed, Scopus, and Embase) were screened for eligible scientific reports. The key words "facial pain" or "trigeminal" or "anaesthesia dolorosa" and "motor cortex stimulation" were used in any possible combination. The last search was launched in April 2020. Two reviewers (A.R. and E.I.) independently screened the abstracts and the references list. Any difference was solved by consensus with a third senior author (N.M.).

Studies were included if they met all the following criteria: (1) prospective or retrospective studies on MCS in patients with FP in English language, (2) series with $>5$ patients, and (3) series which clinical data, outcome, and follow-up (FU) were reported for each patient. As outcome indicators, we studied the implantation rate (the rate of patients implanted after the stimulation trial), the responder rate after MCS at latest FU, the tolerance rate (if reported in the studies for each patient or otherwise evaluated as an increase of $>$ two points on VAS scale during FU) and the changes in visual analogue scale (preoperatively and at latest FU). Moreover, we studied the influence of sex, age ( $<50$ vs. $\geq 50$ years), FP syndrome (central vs. peripheral), pain duration before MCS, and history of previous operations on the clinical outcomes with univariate and multivariate analysis (logistic regression model).

Statistical Analysis

Statistical analyses were done using StatView version 5 software (SAS Institute Inc.). Statistical comparison of continuous variables was performed by Student's $t$ test. Statistical comparison

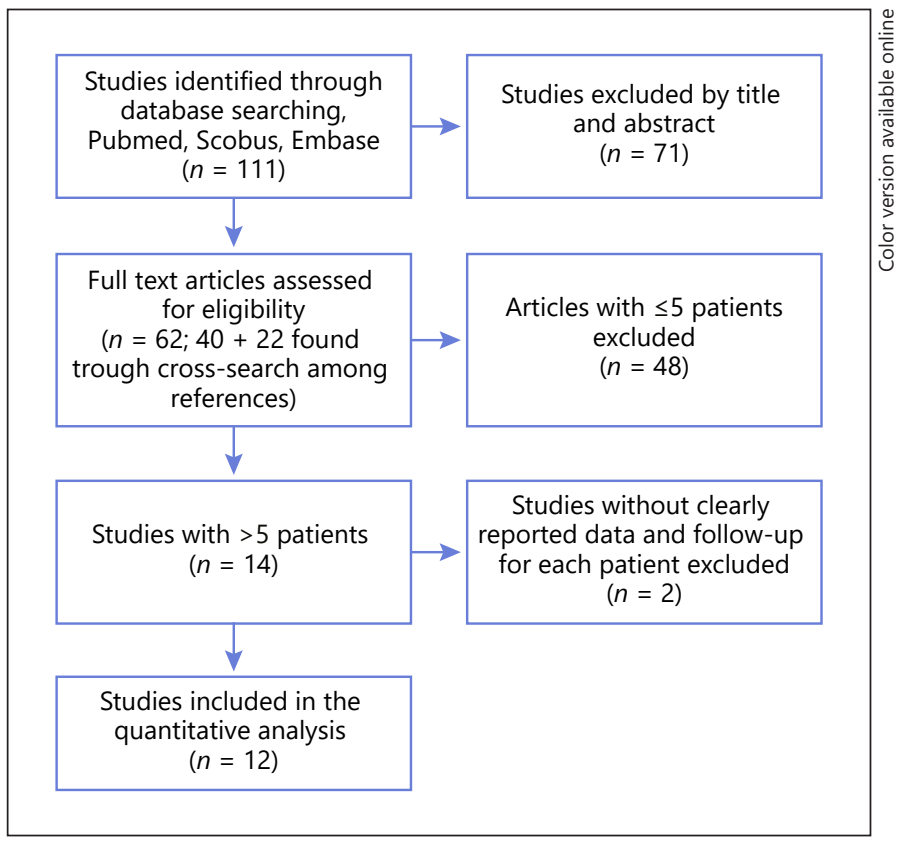

Fig. 1. Flowchart of study selection.

of categorical variables was performed by $\chi^{2}$ statistic, using the Fisher exact test. A multivariate logistic regression model was used to estimate the odds ratio to be a patient implanted and to be a responder, while adjusting for baseline variables that included sex, age, FP syndrome, and pain duration before MCS. Differences were considered significant at $p<0.05$.

\section{Results}

A total of 111 articles were identified and reviewed (Fig. 1). Finally, 12 studies were included in the present investigation (Table 1) [7, 9, 20-29] for a total of 108 patients. A summary of demographic and clinical data of patients included in the pooled analysis is reported in Table 2. Before MCS definitive implantation, all patients were submitted to a stimulation trial. Out of them, 96/108 $(88.88 \%)$ were submitted to permanent MCS. The mean age at implantation was $55.04 \pm 13.03$ years, and the mean FU was $41.89 \pm 42.86$ months. The mean duration of pain before MCS implantation was $5.92 \pm 4.72$ years. Twentyfour patients had a central FP syndrome, and 84 had a peripheral FP syndrome. Overall, at latest FU there were $68 / 96$ (70.83\%) patients who responded to MCS. We were able to study the impact of the following factors for the univariate and multivariate analysis in this amount of patients: sex, $n=82$; age, $n=82$; FP syndrome type, $n=108$; pain duration before MCS, $n=58$; and history of previous 
Table 1. Studies included in the systematic review on MCS in FP

\begin{tabular}{llcc}
\hline Author & Type of article & Patients, $n$ & FU, months \\
\hline Meyerson et al. [9] & Retrospective & 5 & 14.4 \\
Herregodts et al. [20] & Retrospective & 7 & 12.7 \\
Ebel et al. [21] & Retrospective & 7 & 14.5 \\
Nguyen et al. [7] & Retrospective & 7 & 28.3 \\
Brown et al. [22] & Retrospective & 10 & 10 \\
Rasche et al. [23]* & Retrospective & 7 & 49.7 \\
Pirotte et al. [24] & Retrospective & 7 & 22.4 \\
Lefauceheur et al. [25] & Retrospective & 6 & 12 \\
Fagundes-Pereyra et al. [26] & Retrospective & 6 & 29.1 \\
Raslan et al. [27] & Retrospective & 11 & 33 \\
Kolodziej et al. [28] & Retrospective & 9 & $6-72(39$ mean) \\
Rasche et al. [29] & Retrospective & 26 & 67.2 \\
\hline
\end{tabular}

* In this study, patients with peripheral FP were not considered because the long-term results of these patients have been reported in Rasche et al. [29]. MCS, motor cortex stimulation; FP, facial pain; FU, follow-up; FP, facial pain.

operations, $n=50$. Amongst the studied factors, the younger age $(p=0.0478)$ and a peripheral FP syndrome $(p=0.0006)$ positively affected the definitive implantation rate on univariate analysis. None of the studied factors affected the probability to be a responder. Tolerance to the MCS was developed in 23/66 (34.84\%) of cases and none of the studied factors affected the possibility to develop a tolerance after MCS. When studied (41 patients), the preoperative VAS significantly decreased at latest FU $(8.83 \pm 1.17$ and $4.31 \pm 2.05$, respectively; $p<0.0001)$. In the multivariate analysis, younger age emerged as a factor strongly associated to a higher probability to go to a definitive MCS implant ( $p=0.0415$, Table 3 ).

\section{Discussion}

The idea that the motor cortex could be implied in pain modulation is not new and dates back to the last century when Penfield and Jasper discovered that motor cortex excision during epilepsy surgery was related to the relief from some types of severe pain, obviously followed by serious morbidities [30]. Moreover they noted that the sensory cortex excision did not accomplish the same goal [30]. From a pathophysiological point of view, the activation of the connection fibres amongst motor and sensory cortex had been initially proposed by Tsubokawa et al. [6] as the main mechanism responsible for the implementation of the nociceptive inhibitory system at the thalamus level. However, different studies suggest that MCS would be able to modulate nociception also at the level of
Table 2. Demographic and clinical data of patients included in the pooled analysis

\begin{tabular}{lc}
\hline Patients & 108 \\
Sex (M/F)* & $26 / 56$ \\
Mean age, years* & $55.04 \pm 13.03$ \\
Mean FU, months & $41.89 \pm 42.86$ \\
\hline Type of pain & \\
Central pain & 24 \\
Brainstem infarction & 2 \\
Central demielynation & 1 \\
Post-stroke pain & 14 \\
Other causes & 7 \\
Peripheral pain & 84 \\
Trigeminal NP** & 68 \\
Typical drug-resistant trigeminal neuralgia & 10 \\
Persistent idiopathic FP & 2 \\
Other causes & 4 \\
Patients with previous operations (yes/not)*** & $25 / 25$ \\
\hline
\end{tabular}

Number of previous operations

Patients with 1 previous operation $\quad 42$

Patients with 2 previous operations $\quad 14$

Patients with 3 previous operations $\quad 7$

Patients with 4 previous operations 2

Type of previous operations 2

Posterior fossa surgery 18

Percutaneous balloon compression $\quad 1$

Percutaneous glycerol injection 3

Radiofrequency rhizotomy $\quad 10$

Gamma knife surgery 3

$\begin{array}{ll}\text { Previous other site neuromodulation } & 7\end{array}$

FU, follow-up; NP, neuropathic pain; FP, facial pain. * available in 82 cases; ${ }^{* *}$ including anaesthesia dolorosa, trigeminal post-herpetic and post-traumatic pain; *** available in 50 cases. 
Table 3. Logistic model coefficients table for MCS implant

\begin{tabular}{lcccccrrr}
\hline & Coef & Std. error & Coefficient/SE & $\chi^{2}$ & $p$ value & Exp (coef) & 95\% lower & 95\% upper \\
\hline Y: constant & 3.206 & 1.760 & 1.822 & 3.319 & 0.0685 & 24.675 & 0.784 \\
Pain duration before MCS & 0.088 & 0.087 & 1.007 & 1.013 & 0.3141 & 1.092 & 0.920 \\
FP syndrome & 0.95 & 0.814 & 1.167 & 1.362 & 0.2431 & 2.585 & 0.525 & 1.506 \\
Sex & 0.520 & 0.898 & 0.579 & 0.335 & 0.5626 & 1.682 & 0.289 \\
Age & -0.053 & 0.026 & -2.039 & 4.156 & 0.0415 & 0.948 & 0.901 \\
\hline
\end{tabular}

MCS, motor cortex stimulation; FP, facial pain.

brainstem, cingulate gyrus, anterior insula, orbitofrontal cortex, and dorsal horns of the spinal cord [31]. Recent studies on animal models of NP support the hypothesis that MCS would be effective on chronic pain because of the activation of protein kinase $\mathrm{M}$ zeta, a regulator of synaptic plasticity, in the anterior cingulate cortex [32]. From a technical point of view, MCS is performed under general anaesthesia by a small craniotomy even if the use of burr hole techniques under local anaesthesia has also been reported $[6,9]$. The current technique implies the positioning of a 4- or 8-polar electrode paddle in the epidural space, and the identification of precentral sulcus by the presence of N20-P20 phase reversal on median nerve somatosensory evoked potentials. After confirmation of the electrodes' correct position through motor evoked potential by electromyography, the paddle is sutured to the dura. Recently, the identification of the motor strip on a preoperative functional MRI has been reported as a useful tool during surgery [24]. The subdural electrodes placement has been considered but this strategy was associated to a higher risk of complications (subdural haematoma and epileptic seizures) without raising the effectiveness [33]. To our knowledge, this was the first systematic review with a pooled statistical analysis which assessed effectiveness of MCS in FP investigating some predictive factors of positive response. Our pooled analysis confirmed the efficacy of MCS in treating FP without a significant difference amongst the different causes. Furthermore, we found a significant reduction of the VAS values at last FU whenever reported. Some authors suggested that MCS would be more efficacious in peripheral NP over central NP [17]; however, our analysis fails to show such a difference. These data could be explained by the fact that in all included studies of our pooled analysis, a stimulation trial had been performed. In fact, we found that peripheral FP positively affects the possibility to prosecute with full implantation after the trial. Thus, pa- tients with central FP could have decided to not go on with the definitive implantation due to the lack of response to the stimulation trial. Some authors supported the TMS of the motor cortex in order to identify patients who will be responders to MCS, avoiding the more invasive neurostimulation trial [34-36]. While the positive predictive value of this test has been accepted, the negative predictive value of TMS is still debated [36]. Thus, it is uncertain if TMS should be routinely used to rule out patients for MCS. Another strong predictive factor for definitive implantation in our pooled analysis was the younger age of MCS. In our opinion, this may be explained by the fact that younger patients could be more compliant during the trial phase of this advanced technology. Moreover, a more efficient synaptic plasticity, which is typical of younger patients, could play a role in the better results in this category of patients. Thus, MCS might be proposed earlier in the clinical history of patient $[2,3]$. Nonetheless in our pooled analysis we found that the duration of pain does not affect the possibility to respond to MCS. This could be related to the fact that MCS induces a brain plasticity which is probably not influenced by the molecular mechanisms underlying the NP maintenance [31, 32]. Thus, also patients with longer pain history should not be excluded a priori from this approach. Even if we found no factors associated with the tolerance development, some authors reported tolerance episodes along time $[6,7,21]$. The mechanism of this phenomenon is still not completely understood. Further studies are needed to better understand if the stimulation modalities and parameters could affect the tolerance development to MCS. In our analysis, the most frequent complication was the occurrence of transient intraoperative seizures. Clinically important issues such as epidural haematoma or stroke were occasionally reported $[7,9,21$, $23,24,28,29]$. Our study has some limitations. All articles included in our systematic review were retrospective. The 
mean FU greatly varied amongst the studies, and it was $\geq 5$ years only in few cases.

\section{Conclusion}

This systematic review evidenced the effectiveness of MCS in treating FP. In our pooled analysis, younger age emerged as a positive prognostic factor for definitive implantation. Further studies with longer FU are needed to better evaluate the long-term results of MCS.

\section{Statement of Ethics}

The paper is exempt from Ethics Committee approval because it is a systematic literature review.

\section{Conflicts of Interest Statement}

The authors have no conflicts of interest to declare.

\section{Funding Sources}

No funding received for this study.

\section{Author Contributions}

Acquisition, analysis, or interpretation of data: Alessandro Rapisarda, Eleonora Ioannoni, and Nicola Montano; drafting and revising the work: Nicola Montano, Eleonora Ioannoni, and Alessandro Izzo; final approval of the version to be published: Alessandro Rapisarda and Nicola Montano.

\section{References}

1 Colloca L, Ludman T, Bouhassira D, Baron R, Dickenson AH, Yarnitsky D, et al. Neuropathic pain. Nat Rev Dis Primers. 2017;3:17002.

2 Cruccu G, Aziz TZ, Garcia-Larrea L, Hansson $P$, Jensen TS, Lefaucheur JP, et al. EFNS guidelines on neurostimulation therapy for neuropathic pain. Eur J Neurol. 2007 Sep; 14(9):952-70.

3 O'Connor AB, Dworkin RH. Treatment of neuropathic pain: an overview of recent guidelines. Am J Med 2009 Oct;122(10 Suppl l):S22-32.

4 Zakrzewska JM. Differential diagnosis of facial pain and guidelines for management. $\mathrm{Br} \mathrm{J}$ Anaesth. 2013;111(1):95-104.

5 Benoliel R, Gaul C. Persistent idiopathic facial pain. Cephalalgia. 2017 Jun;37(7):680-91.

6 Tsubokawa T, Katayama Y, Yamamoto T, Hirayama $\mathrm{T}$, Koyama $\mathrm{S}$. Chronic motor cortex stimulation for the treatment of central pain. Acta Neurochir Suppl. 1991;52:137-9.

7 Nguyen JP, Keravel Y, Feve A, Uchiyama T, Cesaro P, le Guerinel C, et al. Treatment of deafferentation pain by chronic stimulation of the motor cortex: report of a series of 20 cases. Acta Neurochir Suppl. 1997;68:54-60.

8 Tsubokawa T, Katayama Y, Yamamoto T, Hirayama $\mathrm{T}$, Koyama $\mathrm{S}$. Chronic motor cortex stimulation in patients with thalamic pain. J Neurosurg. 1993 Mar;78(3):393-401.

9 Meyerson BA, Lindblom U, Linderoth B, Lind G, Herregodts P. Motor cortex stimulation as treatment of trigeminal neuropathic pain. Acta Neurochir Suppl. 1993;58:150-3.

10 Esfahani DR, Pisansky MT, Dafer RM, Anderson DE. Motor cortex stimulation: Functional magnetic resonance imaging-localized treatment for three sources of intractable facial pain: Report of 3 cases. J Neurosurg. 2011 Jan;114(1):189-95.
11 Tanei T, Kajita Y, Wakabayashi T. Motor cortex stimulation for intractable neuropathic facial pain related to multiple sclerosis. Neurol Med Chir. 2010;50(7):604-7.

12 Saitoh Y, Shibata M, Sanada Y, Mashimo T. Motor cortex stimulation for phantom limb pain. Lancet. 1999;353(9148):212.

13 Nuti C, Peyron R, Garcia-Larrea L, Brunon J, Laurent B, Sindou M, et al. Motor cortex stimulation for refractory neuropathic pain: four year outcome and predictors of efficacy. Pain. 2005 Nov;118(1-2):43-52.

14 Ali M, Saitoh Y, Oshino S, Hosomi K, Kishima $\mathrm{H}$, Morris S, et al. Differential efficacy of electric motor cortex stimulation and lesioning of the dorsal root entry zone for continuous vs paroxysmal pain after brachial plexus avulsion. Neurosurgery. 2011 May;68(5):1252-8.

15 Louppe JM, Nguyen JP, Robert R, Buffenoir $\mathrm{K}$, de Chauvigny E, Riant T, et al. Motor cortex stimulation in refractory pelvic and perineal pain: Report of two successful cases. Neurourol Urodyn. 2013 Jan;32(1):53-7.

16 Fonoff ET, Hamani C, Ciampi De Andrade D, Yeng LT, Marcolin MA, Jacobsen Teixeira M. Pain relief and functional recovery in patients with complex regional pain syndrome after motor cortex stimulation. Stereotact Funct Neurosurg. 2011 Jun;89(3):167-72.

17 Fontaine D, Hamani C, Lozano A. Efficacy and safety of motor cortex stimulation for chronic neuropathic pain: critical review of the literature-clinical article. J Neurosurg. 2009 Feb;110(2):251-6.

18 Lima MC, Fregni F. Motor cortex stimulation for chronic pain: systematic review and metaanalysis of the literature. Neurology. 2008 Jun 10;70(24):2329-37.
19 Moher D, Liberati A, Tetzlaff J, Altman DG, Altman D, Antes G, et al. Preferred reporting items for systematic reviews and meta-analyses: the PRISMA statement. PLoS Med. 2009 Jul;6(7):e1000097.

20 Herregodts P, Stadnik T, De Ridder F, D'Haens J. Cortical stimulation for central neuropathic pain: 3-D surface MRI for easy determination of the motor cortex. Acta Neurochir Suppl. 1995;64:132-5.

21 Ebel H, Rust D, Tronnier V, Böker D, Kunze $\mathrm{S}$. Chronic precentral stimulation in trigeminal neuropathic pain. Acta Neurochir. 1996; 138(11):1300-6.

22 Brown JA, Pilitsis JG. Motor cortex stimulation for central and neuropathic facial pain: A prospective study of 10 patients and observations of enhanced sensory and motor function during stimulation. Neurosurgery. 2005 Feb;56(2):290-7.

23 Rasche D, Ruppolt M, Stippich C, Unterberg A, Tronnier VM. Motor cortex stimulation for long-term relief of chronic neuropathic pain: a 10 year experience. Pain. 2006 Mar; 121(1-2):43-52.

24 Pirotte B, Voordecker P, Neugroschl C, Baleriaux $\mathrm{D}$, Wikler $\mathrm{D}$, Metens $\mathrm{T}$, et al. Combination of functional magnetic resonance imaging-guided neuronavigation and intraoperative cortical brain mapping improves targeting of motor cortex stimulation in neuropathic pain. Neurosurgery. 2008 Jun;62(6 Suppl 3):941.

25 Lefaucheur JP, Drouot X, Cunin P, Bruckert $\mathrm{R}$, Lepetit $\mathrm{H}$, Créange A, et al. Motor cortex stimulation for the treatment of refractory peripheral neuropathic pain. Brain. 2009 Jun; 132(Pt 6):1463-71. 
26 Fagundes-Pereyra WJ, Teixeira MJ, Reyns N, Touzet G, Dantas S, Laureau E, et al. Motor cortex electric stimulation for the treatment of neuropathic pain. Arq Neuropsiquiatr. 2010 Dec;68(6):923-9.

27 Raslan AM, Nasseri M, Bahgat D, Abdu E, Burchiel KJ. Motor cortex stimulation for trigeminal neuropathic or deafferentation pain: An institutional case series experience. Stereotact Funct Neurosurg. 2011;89(2):838.

28 Kolodziej MA, Hellwig D, Nimsky C, Benes L. Treatment of central deafferentation and trigeminal neuropathic pain by motor cortex stimulation: report of a series of 20 patients. J Neurol Surg A Cent Eur Neurosurg. 2015 Sep; 77(1):52-8.
29 Rasche D, Tronnier VM. Clinical significance of invasive motor cortex stimulation for trigeminal facial neuropathic pain syndromes. Neurosurgery. 2016 Nov;79(5):655-66.

30 Penfield W, Jasper H. Epilepsy and the functional anatomy of the human brain. Oxford, England: Little, Brown \& Co.; 1954.

31 Cioni B, Meglio M, Perotti V, de Bonis P, Montano N. Neurophysiological aspects of chronic motor cortex stimulation. Neurophysiol Clin. 2007 Dec;37(6):441-7.

32 Cha M, Um SW, Kwon M, Nam TS, Lee BH. Repetitive motor cortex stimulation reinforces the pain modulation circuits of peripheral neuropathic pain. Sci Rep. 2017 Dec;7(1): 7986.

33 Monsalve GA. Motor cortex stimulation for facial chronic neuropathic pain: A review of the literature. Surg Neurol Int. 2012 Oct; 3(Suppl 4):S290.
34 Zhang X, Hu Y, Tao W, Zhu H, Xiao D, Li Y. The effect of motor cortex stimulation on central poststroke pain in a series of 16 patients with a mean follow-up of 28 months. Neuromodulation. 2017;20(5):492-6.

35 André-Obadia N, Peyron R, Mertens P, Mauguière F, Laurent B, Garcia-Larrea L. Transcranial magnetic stimulation for pain control. Double-blind study of different frequencies against placebo, and correlation with motor cortex stimulation efficacy. Clin Neurophysiol. 2006 Jul;117(7):153644.

36 Lefaucheur JP, Ménard-Lefaucheur I, Goujon C, Keravel Y, Nguyen JP. Predictive value of rTMS in the identification of responders to epidural motor cortex stimulation therapy for pain. J Pain. 2011 Oct;12(10):1102-11. 